\title{
The effect of counselling, graded exercise and usual care for people with chronic fatigue in primary care: a randomized trial
}

\author{
L. Ridsdale ${ }^{1 *}$, M. Hurley ${ }^{1}$, M. King ${ }^{2}$, P. McCrone ${ }^{1}$ and N. Donaldson ${ }^{3}$ \\ ${ }^{1}$ Department of Clinical Neuroscience, King's College London, Institute of Psychiatry, Academic Neuroscience Centre, London, UK \\ ${ }^{2}$ Royal Free and University College Hospital, London, UK \\ ${ }^{3}$ King's College London Dental Institute, London, UK
}

Background. To evaluate the effectiveness of graded exercise therapy (GET), counselling (COUNS) and usual care plus a cognitive behaviour therapy (CBT) booklet (BUC) for people presenting with chronic fatigue in primary care.

Method. A randomized controlled trial in general practice. The main outcome measure was the change in the Chalder fatigue score between baseline and 6 months. Secondary outcomes included a measure of global outcome, including anxiety and depression, functional impairment and satisfaction.

Results. The reduction in mean Chalder fatigue score at 6 months was 8.1 [95\% confidence interval (CI) 6.6-10.4] for BUC, 10.1 (95\% CI 7.5-12.6) for GET and 8.6 (95\% CI 6.5-10.8) for COUNS. There were no significant differences in change scores between the three groups at the 6- or 12-month assessment. Dissatisfaction with care was high. In relation to the BUC group, the odds of dissatisfaction at the 12-month assessment were less for the GET [odds ratio (OR) $0.11,95 \%$ CI 0.02-0.54, $p=0.01$ ] and COUNS groups (OR 0.13, 95\% CI 0.03-0.53, $p=0.004$ ).

\begin{abstract}
Conclusions. Our evidence suggests that fatigue presented to general practitioners (GPs) tends to remit over 6 months to a greater extent than found previously. Compared to BUC, those treated with graded exercise or counselling therapies were not significantly better with respect to the primary fatigue outcome, although they were less dissatisfied at 1 year. This evidence is generalizable nationally and internationally. We suggest that GPs ask patients to return at 6 months if their fatigue does not remit, when therapy options can be discussed further.
\end{abstract}

Received 10 May 2011; Revised 25 January 2012; Accepted 25 January 2012 ; First published online 28 February 2012

Key words: Chronic fatigue, counselling, graded exercise, primary care, randomized trial, therapy.

\section{Introduction}

Graded exercise therapy (GET) or cognitive behaviour therapy (CBT) can be moderately effective for chronic fatigue syndrome (CFS) when provided by highly experienced therapists in secondary care (Whiting et al. 2001; NICE, 2007), but there is little evidence in primary care. Symptoms of fatigue are common (Wessely et al. 1997) among patients seeking care from general practitioners (GPs). When no specific cause is identified, GPs do not distinguish specifically between fatigue that does or does not meet criteria for CFS (Darbishire et al. 2003). This diagnosis requires 3 or 6 months' duration, $50 \%$ disability, and other symptoms (Hickie et al. 2009). In a cohort study we found that, without treatment, about $60 \%$ of patients with fatigue

* Address for correspondence: Dr L. Ridsdale, Department of Clinical Neuroscience, King's College London, Institute of Psychiatry, Academic Neuroscience Centre, PO41, De Crespigny Park, Denmark Hill Campus, London SE5 8AF, UK.

(Email : leone.ridsdale@kcl.ac.uk) continued to have case-level symptoms 6 months later (Ridsdale et al. 1993). Disability associated with fatigue imposes considerable cost on patients and their families (Sabes-Figuera et al. 2010).

In prior trials, CBT, counselling and GET were associated with reduced fatigue in primary care 6 months later (Ridsdale et al. 2001, 2004). However, these previous trials did not include a control group, so it is unclear whether the reduction was a time effect. When we compared CBT to counselling, the key predictor of a good fatigue outcome was the patients' emotional processing, including the expression, acknowledgement and acceptance of emotional distress, and specific ingredients of a particular therapy were not associated with helping patients to change (Godfrey et al. 2007). It remains unclear whether, compared to usual care, therapies delivered in primary care can reduce symptoms and, if they do, whether the benefit lasts longer than 6 months.

The aim of this trial was to compare graded exercise and counselling, both of which are readily available in

The online version of this article is published within an Open Access environment subject to the conditions of the Creative Commons Attribution-NonCommercial-ShareAlike licence <http://creativecommons.org/licenses/by-nc-sa/2.5/>. The written permission of Cambridge University Press must be obtained for commercial re-use. 
Table 1. Inclusion and exclusion criteria for the study

Inclusion criteria

1. Age $16-75$ years inclusive

2. Fatigue of 3 months or more

3. Patient presents fatigue as a main/important problem

4. Patient may be on stable drug regime for physical and/or psychological problems

5. Patient has had a normal full blood count, erythrocyte sedimentation rate (ESR) and thyroid function test during the 6 months prior to entering the study, or on entry to the study

\section{Exclusion criteria}

1. A score of $<4$ on a fatigue scale

2. Patient has a physical condition/problem that causes, or is likely to cause, fatigue

3. Patient is suffering from psychotic illness, organic brain syndrome, or substance dependency

4. Patient is currently receiving treatment from a psychiatrist, counsellor, psychologist, community psychiatric nurse (CPN), physiotherapist, or other exercise specialist

5. Patient is unable to come to the surgery for the treatment intervention

6. Patient has severe asthma, chronic obstructive airway disease and/or ischaemic heart disease that would contraindicate graded exercise

general practice, to usual primary care plus a booklet on CBT (BUC). The purpose of introducing a booklet was to encourage GPs to refer, and patients to participate. A booklet has been shown to slightly enhance usual care in a community-based sample (Chalder et al. 1997). The trial objective was to evaluate changes in fatigue scores between patients in the three trial arms. Our null hypothesis was for no difference between the groups. Secondary outcomes included anxiety and depression, work/social adjustment, and patient satisfaction.

\section{Method}

Participants aged 16 to 75 years who had consulted their GP complaining of fatigue for more than 3 months and met the eligibility criteria for the trial (Table 1) were recruited between November 2003 and October 2007 from general practices in London and South East England. The eligible population was 236000. GPs asked patients to give informed written consent for a meeting with a research associate at the practice. At this interview the trial was fully explained and written consent obtained. The trial had multicentre ethics committee approval (MREC West Midlands MREC/02/7/71).
The study was a three-armed, randomized trial comparing GET or counselling (COUNS) with BUC. Patients were assessed at baseline, and at 6 and 12 months. The unit of randomization was the patient, the allocation ratio was $1: 1: 1$, and block randomization (blocks of size 9) was used. A statistician working in a separate site performed the randomization and opaque sealed envelopes bearing sequential ID numbers were prepared, containing the randomly allocated treatment codes: BUC, GET or COUNS. The research assistant took an envelope in sequence to each baseline assessment, opened the envelope in front of the patient and explained the treatment assignment. At 6 and 12 months, each patient was sent a questionnaire and asked to complete it at home and send it to the research assistant.

Participants were offered eight sessions of individual treatment by a therapist trained in graded exercise or counselling at 2-week intervals at their local primary care practice, followed by two telephone calls from the therapist 1 month apart.

\section{Graded exercise}

GET is based on the principles of exercise prescription devised by the American College of Sports Medicine (2000). It consisted of supervised exercise, adapted to each patient's current physical capacity, that is gradually increasing in duration according to a protocol designed for patients with CFS (Fulcher \& White, 1998). It has been evaluated in previous trials by this research group (Ridsdale et al. 2004). Registered physiotherapists taught participants how to measure their heart rate and provided a tailored walking exercise programme to each individual that built up from an initial 5-30 min of exercise a day. Patients were advised not to exceed the recommended duration or intensity of exercise. Exercise took the form of walking and each patient was given an instruction manual and recording sheet to monitor their progress. Eight physiotherapists saw a mean number of 10 patients for 30-min sessions.

\section{Counselling}

The counselling style used in this study followed the Rogerian client-centred, non-directive format that encouraged the patient to talk through difficulties, and reflect on their experiences and thoughts so as to understand themselves better, to arrive at alternative understandings, to uncover the links between current distress and past experience, and to provide the conditions for growth and healing (Godfrey et al. 2007). Patients were offered 50-min sessions. A senior counsellor provided supervision. Eight British 
Association for Counselling and Psychotherapy (BACP)-registered therapists saw a mean number of 9.5 patients each.

\section{Usual care}

Participants in the comparison arm received usual care treatment from their family doctors. To encourage practice and patient participation, this was coupled with a booklet describing causes of fatigue and providing self-help techniques based on CBT principles (Chalder, 1995).

Fatigue was assessed using the Chalder fatigue scale (Chalder et al. 1993), an 11-item questionnaire that, when Likert scored $(0,1,2,3)$, produces a total score between 0 and 33 . The primary outcome measure was the change in the Chalder fatigue score between baseline and 6 months. Secondary outcomes included the Hospital Anxiety and Depression Scale (HADS; Zigmond \& Snaith, 1983) and the Work and Social Adjustment Scale (WSAS; Ware et al. 1992), measuring degree of functional impairment in work, home, private and leisure (not at all impaired $=0$; very severely impaired $=8$ ). Patient satisfaction with treatment was recorded at 6 and 12 months using seven categories, but given the sparse cross-tabulations by therapy, the categories were collapsed into three (very/moderately/slightly satisfied; neither; very/ moderately/slightly dissatisfied).

At baseline, self-reported rating using the European Questionnaire measuring health-related quality of life in five dimensions with UK weights attached (EQ-5D; Dolan et al. 1995) was measured, in addition to patient's preferences for treatment and other characteristics, which were measured serially.

\section{Statistical analysis}

We based our power calculation on previous studies where the mean changes in fatigue score at 6 months were approximately 5 for usual medical care, 10 for GET and 9 for counselling (Ridsdale et al. 1993, 2001, 2004). In this trial, we aimed to detect a difference of 4 units (standard deviation of 5.5) in the mean change fatigue score between usual care and either of the active interventions (GET or COUNS), at the 5\% (twotailed) level of significance and $80 \%$ power, for which 30 patients per arm were required. With 15 patients, on average, for each therapist and an intra-therapist correlation of $5 \%$ (design effect $=1.7$ ), a total of 153 patients across 18 therapists were required. Assuming a drop-out rate of $30 \%$, a total of 225 patients were sought.

An intention-to-treat (ITT) approach was used. The principal analysis consisted of mixed linear, ordinal and logistic regressions for the continuous, ordinal and binary outcomes respectively. As the study is a randomized trial of moderately large sample size and with no anticipated confounders, the unadjusted comparison of the groups is presented for both the primary and the secondary analysis. We nevertheless checked all the potential prognostic variables assessed in the study: demographic, social and clinical baseline characteristics (as shown in Tables 2 and 3). The multiple regressions included only those variables that were significant at $<0.25$ in the univariate models. Final significance in the multiple regressions was judged at the usual 5\% level. A binary variable was created to indicate whether a patient had been allocated to a therapy that would not have been of their choice. We explored the effect of this on outcome and adherence.

Classification of missing data, using missing at random (MAR) analysis, was inferred by modelling the likelihood of missingness using a logistic regression model and we modelled the outcome using both complete case and multiple imputations for the missing values of the independent and dependent variables. The multiple imputation algorithm generated 20 datasets assuming a greater than $95 \%$ fraction of missing information, 50 times per data set, and the imputation models were based on results from complete case analyses. Moreover, to account for variables found to be related to missingness in the MAR analysis, the variables 'age' and 'compliance status' were also included in the imputation models. Analyses of the 20 datasets was conducted in accordance with Rubin's rules (Rubin, 1987). In addition, using all available data, estimates of means for the complier and non-complier groups within each therapy are provided. The data analysis was conducted in Stata version 11 (Stata Corporation, USA).

\section{Results}

GPs referred 324 patients with fatigue to the study, and 222 reached baseline assessment (Fig. 1). Adherence to the allocated therapy was measured by the number of therapy sessions attended. These ranged from zero to eight, with a mean of 5.8 (S.D. =3) attended for GET and 5.9 (s.D.=2.8) for COUNS. The 25th, 50th (median) and 75th percentiles were 3-8-8 for GET and 4-8-8 for COUNS. Age was the only factor significantly associated with compliance: patients aged over 35 years were 2.6 times as likely to attend all eight sessions compared to younger patients.

The three groups were balanced by sociodemographic characteristics and baseline health outcome measures (Table 2). Table 3 illustrates primary and secondary outcomes at baseline, 6 and 12 months. A total of 222 patients were assessed at baseline, and 
Table 2. Demographic and social characteristics of each group at baseline

\begin{tabular}{lcccc}
\hline & Overall & BUC & GET & COUNS \\
\hline Age (years), mean (range) & $39.8(38.0-41.6)$ & $37.3(34.0-40.0)$ & $42.6(39.0-46.0)$ & $39.7(36.0-43.0)$ \\
Gender (male), $n$ (\%) & $48(22)$ & $15(21)$ & $15(21)$ & $18(24)$ \\
No. of months with fatigue at & $53(44-62)$ & $51(33-70)$ & $57(42-72)$ & $51(36-65)$ \\
presentation, mean (range) & & & & \\
EQ-5D (Euroqol), mean (95\% CI) & $0.64(0.60-0.67)$ & $0.64(0.57-0.71)$ & $0.62(0.54-0.69)$ & $0.65(0.59-0.71)$ \\
Ethnicity (White British), $n(\%)$ & $150(83)$ & $55(89)$ & $45(80)$ & $50(81)$ \\
Have dependants $n(\%)$ & $73(33)$ & $24(32)$ & $24(34)$ & $25(33)$ \\
Have children under 5, $n(\%)$ & $23(10)$ & $7(9)$ & $20(28)$ & $11(14)$ \\
Have children over 5, $n(\%)$ & $60(26)$ & $20(26)$ & $4(6)$ & $18(24)$ \\
Have elderly dependant, $n$ (\%) & $8(4)$ & $24(3)$ & $24.8(4.7)$ & $2(3)$ \\
Chalder fatigue score, mean (s.D.) & $23.4(4.5)$ & $24.8(4.9)$ & & \\
\hline
\end{tabular}

EQ-5D, European Quality of Life Questionnaire - 5 Dimensions; BUC, usual care plus a cognitive behaviour therapy (CBT) booklet; GET, graded exercise therapy ; COUNS, counselling; CI, confidence interval; s.D., standard deviation.

No significant difference between groups was observed.

163 and 174 patients were assessed at 6 and 12 months respectively. The distribution of missing data was not significantly associated with the allocated group for either the 6-month ( $p=0.78$ ) or the 12month $(p=0.36)$ assessment. There was a significant association between older age and missing data on fatigue score at the 12-month assessment [odds ratio (OR) 0.97, 95\% confidence interval (CI) 0.94-1.0, $p=0.02]$.

\section{Fatigue scale}

There were 559 observations in 222 patients recruited by 19 therapists. There was no significant therapist effect. A mixed multiple linear regression on the complete data revealed a significant time effect: there was a mean improvement in the Chalder fatigue scale of 9.2 (mean $=-9.2,95 \%$ CI -10.3 to -8.1 , $p<0.0001$ ) between baseline and the 6-month followup and a non-significant mean improvement of 0.94 (mean $=-0.94,95 \% \mathrm{CI}-1.9$ to $0.03, p=0.06$ ) between the 6-month and the 12-month follow-up. The mean improvement between baseline and 12 months was 10.1 (mean $=-10.1,95 \% \mathrm{CI}-11.2$ to $-9.1, p=0.0004)$. None of the potential prognostic variables tested had a significant effect on outcome. The time by group interaction was not significant, indicating that the reduction in fatigue at either 6 or 12 months was similar for the three groups. The mean improvement at 6 months was 8.1 (95\% CI 6.6-10.4) for BUC, 10.1 (95\% CI 7.5-12.6) for GET and 8.6 (95\% CI 6.5-10.8) for COUNS. The change (reduction) at 12 months was -10.2 ( $95 \%$ CI -12 to -8.3$)$ for BUC, -10.7 (95\% CI -12.8 to -8.6$)$ for GET and $-9.2(95 \% \mathrm{CI}-11.2$ to $-7.2)$ for COUNS.
There was no significant difference in the overall mean level of the Chalder fatigue scale between BUC and GET (mean $=-1.13,95 \% \mathrm{CI}-4.2$ to $1.9, p=0.46$ ) or between BUC and COUNS (mean $=0.36,95 \% \mathrm{CI}$ -2.6 to $3.3, p=0.81$ ) at 6 months (Table 3). Similarly, there was no significant difference in means on the Chalder fatigue scale between BUC and GET (mean $=-0.06,95 \% \mathrm{CI}-1.8$ to $1.6, p=0.94$ ) or between BUC and COUNS (mean $=0.99,95 \% \mathrm{CI}-0.7$ to 2.6, $p=0.24$ ) at 12 months.

There were data on preference for treatment for 168 patients. The effect of this variable was not statistically significant on the outcome of fatigue total score. Preference did not show any association with adherence (attending to all eight sessions of therapy) status.

\section{Anxiety and depression and work and social adjustment}

All three groups experienced a similar reduction in HADS depression and anxiety scores between the baseline and 6-month assessments. No significant differences were identified between BUC and GET and between BUC and COUNS on the WSAS (Table 3).

\section{Dissatisfaction with care}

Over half of all patients reported dissatisfaction with care at 6 months, with no significant difference between the three arms (Table 3). An ordinal logistic regression showed that each extra month in the duration of fatigue reported at baseline increased the odds of a higher level of dissatisfaction by $1 \%$ (OR 1.01, 95\% CI 0.9-1.004, $p=0.01$ ). Dissatisfaction increased for participants allocated to BUC between the 6-month and 12-month follow-up, whereas 
Table 3. Observed outcomes by therapy

\begin{tabular}{|c|c|c|c|}
\hline Outcome & BUC & GET & COUNS \\
\hline \multicolumn{4}{|l|}{ Chalder total score, mean (s.D.) } \\
\hline Baseline & $23.4(4.5)$ & $24.8(4.9)$ & $24.8(4.7)$ \\
\hline 6 months & $15.3(8.0)$ & $14.6(8.5)$ & $16.2(8.2)$ \\
\hline 12 months & $13.8(7.7)$ & $14.5(7.7)$ & $15.2(8.4)$ \\
\hline \multicolumn{4}{|l|}{ At 6 months } \\
\hline Change in Chalder total score & $-8.1(-10.4$ to 6.6$)$ & $-10.1(-12.6$ to 7.5$)$ & $-8.6(-10.8$ to -6.5$)$ \\
\hline Mean difference in relation to BUC $(95 \% \mathrm{CI})$ & & $\begin{aligned}-1.3(-4.2 \text { to } 1.9) \\
p=0.46\end{aligned}$ & $\begin{array}{l}0.36(-2.6 \text { to } 3.3) \\
p=0.81\end{array}$ \\
\hline \multicolumn{4}{|l|}{ At 12 months } \\
\hline Change in Chalder total score & $-10.2(-12$ to -8.3$)$ & $-10.7(-12.8$ to -8.6$)$ & $-9.2(-11.2$ to -7.2$)$ \\
\hline Mean difference in relation to BUC $(95 \% \mathrm{CI})$ & & $\begin{array}{l}-0.06(-1.8 \text { to } 1.6) \\
p=0.94\end{array}$ & $\begin{array}{l}0.99(-0.66 \text { to } 2.6) \\
p=0.24\end{array}$ \\
\hline \multicolumn{4}{|l|}{ HADS - Depression, mean score (S.D.) } \\
\hline Baseline & $7.4(5.7)$ & $8.4(3.8)$ & $7.8(3.7)$ \\
\hline 6 months & $6.2(4.3)$ & $6.5(4.3)$ & $6.9(4.6)$ \\
\hline \multicolumn{4}{|l|}{ HADS - Anxiety, mean score (s.D.) } \\
\hline Baseline & $9.1(4.3)$ & $9.3(4.4)$ & $9.4(4.3)$ \\
\hline 6 months & $9.4(4.0)$ & $8.2(4.0)$ & $9.2(3.9)$ \\
\hline \multicolumn{4}{|l|}{ WSAS, mean score (S.D.) } \\
\hline Baseline & $4.4(2.0)$ & $4.5(1.9)$ & $4.7(1.9)$ \\
\hline 6 months & $3.7(2.3)$ & $3.9(1.9)$ & $4.2(2.1)$ \\
\hline \multicolumn{4}{|c|}{ Global outcome: How satisfied are you with your treatment? $n(\%)$} \\
\hline \multicolumn{4}{|c|}{ At 6 months } \\
\hline Very/moderately/slightly satisfied & $16(21.3)$ & $15(21.1)$ & $16(21)$ \\
\hline Neither & $16(21.3)$ & $13(18.3)$ & $13(17.1)$ \\
\hline Very/moderately/slightly dissatisfied & $43(57.3)$ & $43(60.6)$ & $47(61.8)$ \\
\hline \multicolumn{4}{|l|}{ At 12 months } \\
\hline Very/moderately/slightly satisfied & $16(21.3)$ & $25(35.2)$ & $27(35.5)$ \\
\hline Neither & $10(13.3)$ & $9(12.7)$ & $8(10.5)$ \\
\hline Very/moderately/slightly dissatisfied & $49(65.3)$ & $37(52.1)$ & $41(54.0)$ \\
\hline \multirow{2}{*}{\multicolumn{2}{|c|}{ Dissatisfaction in relation to BUC, OR $(95 \% \mathrm{CI})$}} & $0.11(0.02-0.54)$ & $0.13(0.03-0.53)$ \\
\hline & & $p=0.01$ & $p=0.004$ \\
\hline
\end{tabular}

BUC, Usual care plus a cognitive behaviour therapy (CBT) booklet; GET, graded exercise therapy; COUNS, counselling; HADS, Hospital Anxiety and Depression Scale; WSAS, Work and Social Adjustment Scale; OR, odds ratio; CI, confidence interval; S.D., standard deviation.

satisfaction increased for the participants receiving GET and COUNS. In relation to the BUC group, the OR of dissatisfaction at the 12-month assessment was significantly less for the GET (OR $0.11,95 \%$ CI $0.02-0.54, p=0.01$ ) and COUNS groups (OR 0.13, 95\% CI $0.03-0.53, p=0.004)$.

\section{Discussion}

Following entry to the trial for chronic fatigue, the Chalder fatigue score declined in all three groups over 6 months (BUC -8.1, GET -10.1, COUNS -8.6). The mean improvement in fatigue scores after 6 months did not differ significantly between patients receiving BUC and those receiving GET $(-1.13,95 \%$
CI -4.2 to 1.19$)$ or COUNS $(0.36,95 \% \mathrm{CI}-2.6$ to 3.3$)$. However, at 12 months, groups allocated to GET and counselling were less dissatisfied than those allocated to usual care, suggesting that patients valued therapy.

Our results are conclusively negative with CIs for the comparison of change scores well within the minimum difference of interest assumed when choosing the sample size. Our standard deviation estimates were also confirmed in the trial data. There was good compliance with allocated therapy, with patients taking up six to eight sessions. Our findings suggest that many patients improve substantially in the first 6 months. This factor, time, is likely to explain the improvement suggested in prior trials that used evidence from cohorts as comparators rather than 
Enrolment

Allocation
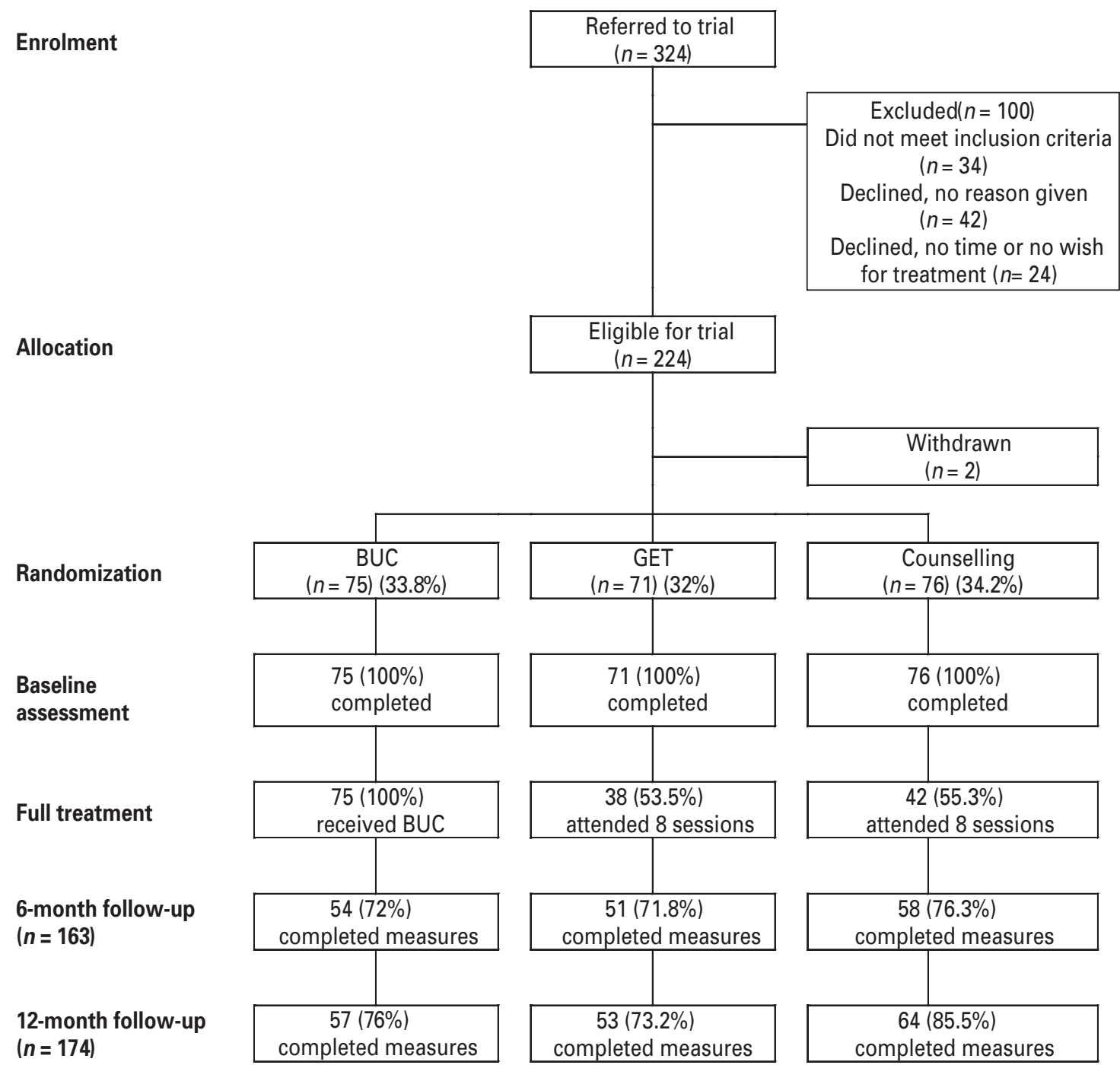

Fig. 1. The flow of participants. BUC, Usual care plus a cognitive behaviour therapy (CBT) booklet; GET, graded exercise therapy.

randomized control groups (Ridsdale et al. 1993, 2001, 2004). GPs manage the majority of patients with fatigue. This evidence derived from primary care is more likely to be generalizable to primary care than evidence derived by therapists working in specialist care with referred CFS patients. A further strength is that we found little evidence of variation in outcome between therapists.

Wearden et al. (2010) published a trial from primary care in which they recruited only patients with more severe CFS. Therapies consisted of pragmatic rehabilitation and supportive listening, implemented by nurses with additional training rather than the physiotherapists and counsellors working in our trial. The nurse interventions also had non-significant effects on fatigue at the 1-year follow-up.

We offered two sessions and two telephone calls more than in our prior trials of six sessions of GET and counselling (Ridsdale et al. 2001, 2004). Several explanations are compatible with the finding of a lack of significant difference in outcome, in particular that greater than expected improvement occurred in the usual care group, and that less improvement occurred in the treatment groups even though up to eight sessions were provided. It is already known that a CBT booklet is itself more effective than usual care (Chalder et al. 1997). A CBT booklet co-intervention with the usual care group may have added slightly to the effectiveness of usual medical care. Most therapists working in usual National Health Service (NHS) practice are less expert, do not see sufficient case volume, or give a therapy long enough to achieve the effect demonstrated in tertiary care trials. In the study by Deale et al. (1997), delivering an average of $15 \mathrm{~h}$ of CBT to participants with CFS achieved a 6-point reduction in mean fatigue 
(binary scored 0011). When CBT was delivered by many NHS CBT therapists in the same centre, but outside the trial context, Quarmby et al. (2007) found that the fatigue score declined by only 3.5 (binary) points. This is less than the mean 4.6 (binary scored) decline we found in our trial for the BUC group.

A recent study by White et al. (2011) of patients with CFS found a moderate difference (mean 3.2 points on a Likert scale) between a longer course (up to 15 sessions) of GET plus usual specialist care after 1 year. Chronic fatigue is a remitting and relapsing condition clinically, and it may be that a longer course of treatment or a booster dose is needed to achieve sustained improvement (Deale et al. 1997; Fulcher \& White, 1997).

The results of the current study do not support early implementation of a short course of either GET or counselling for chronic fatigue in primary care. However, participants tended to be less dissatisfied after graded exercise and counselling. Hitherto, patients with chronic fatigue have expressed dissatisfaction with management (NICE, 2007), and this is supported by our evidence. We found patients who had experienced prolonged fatigue symptoms before the trial were more likely to report dissatisfaction. Dissatisfaction sometimes undermines the doctor-patient relationship (Bowie, 2008). Our quantitative and qualitative studies suggest that patients are more satisfied with seeing a therapist in addition to usual primary care, and qualitative data show that, irrespective of changes in fatigue, both the relationship and time spent with therapists were valued (Godfrey et al., unpublished observations). Satisfaction with primary care is a performance indicator, and eligible for remuneration (Department of Health, 2009). We believe that people appreciate being seen by someone for six to eight sessions of therapy. It may be that time and personal attention are important process variables. It seems likely that, when not offered a therapeutic intervention, dissatisfaction persists or increases. In the absence of evidence for efficacy, GPs can at the least provide usual advice plus written self-help information on managing chronic fatigue, as a form of health promotion in primary care. In a separate paper we report the cost of therapy, and show that GET is less expensive to provide than counselling (Sabes-Figuera et al., unpublished observations).

From the current evidence, we propose that after assessment of patients who present with fatigue in primary care, doctors offer to reassess them in 6 months. If fatigue symptoms persist, the practitioner and patient may discuss further therapy options.

\section{Acknowledgements}

We thank A. Passalacqua, S. Spector, A. Wearden, R. Jones, and our anonymous referees for advice, and the Wellcome Trust for funding (068474/Z/02/Z). We also thank the GPs and practices that participated at the study: Brigstock Medical Centre, Canbury Medical Centre, Cheam Family Practice, Crown Dale Medical Centre, Dr Laurence \& Partners (West Byfleet Health Centre), Elizabeth House Surgery, Fairhill Medical Practice, Hamsey Green Surgery, Hillview Medical Centre, Lambeth Walk Group Practice, Lavender Hill Group Practice, Morden Hill Surgery, Parishes Bridge (West Byfleet Health Centre), Partkside Group Practice, Paxton Green Health Centre, Selsdon Park Practice, South Lewisham Group Practice, Tadworth Medical Centre, The Church Lane Practice, The Hurley Clinic, The Jenner Practice, The Nunhead Surgery, The Oaklands Practice, The Surgery, Lower Addiscombe Road, The Vanbrugh Group Practice 2000, The Woodcote Group Practice, Waterloo Health Centre, Wells Park, and Wrythe Green Surgery.

\section{Declaration of Interest}

None.

\section{References}

American College of Sports Medicine (2000). Guidelines for Exercise Testing and Prescription, 6th edn. Williams \& Wilkins: London.

Bowie P (2008). Fatigue in primary care: a qualitative study. MPhil thesis, King's College London.

Chalder T (1995). Coping with Chronic Fatigue. Sheldon Press: London.

Chalder T, Berelowitz G, Pawlikowska T, Watts L, Wessely S, Wright D, Wallace EP (1993). Development of a fatigue scale. Journal of Psychosomatic Research 37, 147-153.

Chalder T, Wallace P, Wessely S (1997). Self-help treatment of chronic fatigue in the community: a randomized controlled trial. British Journal of Health Psychology 2, 189-197.

Darbishire L, Ridsdale L, Seed P (2003). Distinguishing patients with chronic fatigue from those with chronic fatigue syndrome: a diagnostic study. British Journal of General Practice 53, 441-445.

Deale A, Chalder T, Marks I, Wessely S (1997). Cognitive behavior therapy for chronic fatigue syndrome: a randomized controlled trial. American Journal of Psychiatry 154, 408-414.

Department of Health (2009). NHS 2010-2015: From Good to Great. Preventative, People-Centred, Productive. The Stationery Office: London, UK.

Dolan P, Gudex C, Kind P, Williams A (1995). A Social Tariff for EuroQol: Results from a UK General Population Survey. Centre for Health Economics, University of York: York. 
Fulcher KY, White PD (1997). Randomised controlled trial of graded exercise in patients with the chronic fatigue syndrome. British Medical Journal 314, 1647-1652.

Fulcher KY, White PD (1998). Chronic fatigue syndrome: a description of graded exercise treatment. Physiotherapy $\mathbf{8 4}$, 223-226.

Godfrey E, Chalder T, Ridsdale L, Seed P, Ogden J (2007). Investigating the active ingredients of cognitive behaviour therapy and counselling for patients with chronic fatigue in primary care: developing a new process measure to assess treatment fidelity and predict outcome. British Journal of Clinical Psychology 46, 253-272.

Hickie I, Davenport T, Vernon SD, Nisenbaum R, Reeves WD, Hadzi-Pavlovic D, Lloyd A ; International Chronic Fatigue Syndrome Study Group (2009). Are chronic fatigue and chronic fatigue syndrome valid clinical entities across countries and healthcare settings? Australian and New Zealand Journal of Psychiatry 43, 25-35.

NICE (2007). Chronic Fatigue Syndrome/Myalgic

Encephalomyelitis (or Encephalopathy): Diagnosis and Management of CFS/ME in Adults and Children. National Institute for Health and Clinical Excellence (NICE) clinical guideline 53. NICE: London, UK.

Quarmby L, Rimes KA, Deale A, Wessely S, Chalder T (2007). Cognitive-behaviour therapy for chronic fatigue syndrome: comparison of outcomes within and outside the confines of a randomised controlled trial. Behaviour Research and Therapy 45, 1085-1094.

Ridsdale L, Darbishire L, Seed PT (2004). Is graded exercise better than cognitive behaviour therapy for fatigue? A UK randomized trial in primary care. Psychological Medicine 34, $37-49$.

Ridsdale L, Evans A, Jerrett W, Mandalia S, Osler K, Vora H (1993). Patients with fatigue in general practice: a prospective study. British Medical Journal 307, 103-106.

Ridsdale L, Godfrey E, Chalder T, Seed P, King M, Wallace P, Wessely S ; the Fatigue Trialists' Group (2001). Chronic fatigue in general practice: is counselling as good as cognitive behaviour therapy? A UK randomised trial. British Journal of General Practice 51, 19-24.
Rubin DB (1987). Multiple Imputation for Nonresponse Surveys. John Wiley \& Sons: Hoboken, NJ.

Sabes-Figuera R, McCrone P, Hurley M, King M, Donaldson AN, Ridsdale L (2010). The hidden cost of chronic fatigue to patients and their families. BMC Health Services Research 10, 56.

Ware JE, Sherbourne CD, Davies AR (1992). Developing and testing the MOS 20-item short-form health survey: a general population approach. In Measuring Functioning and Well-Being: The Medical Outcomes Study Approach (ed. A. L. Stewart and J. E. Ware), pp. 277-290. Duke University Press: Durham, NC.

Wearden AJ, Dowrick C, Chew-Graham C, Bentall RP, Morriss RK, Peters S, Riste L, Richardson G, Lovell K, Dunn G; Fatigue Intervention by Nurses Evaluation (FINE) trial writing group and the FINE trial group (2010). Nurse led, home based self help treatment for patients in primary care with chronic fatigue syndrome: randomised controlled trial. British Medical Journal 340, c1777.

Wessely S, Chalder T, Hirsch S, Wallace P, Wright D (1997). The prevalence and morbidity of chronic fatigue and chronic fatigue syndrome: a prospective primary care study. American Journal of Public Health $\mathbf{8 7}$, 1449-1455.

White PD, Goldsmith KA, Johnson AL, Potts L, Walwyn R, DeCesare JC, Baber HL, Burgess M, Clark LV, Cox DL, Bavinton J, Angus BJ, Murphy G, Murphy M, O'Dowd H, Wilks D, McCrone P, Chalder T, Sharpe M; PACE trial management group (2011). Comparison of adaptive pacing therapy, cognitive behaviour therapy, graded exercise therapy, and specialist medical care for chronic fatigue syndrome (PACE) : a randomised trial. Lancet 377, 823-836.

Whiting P, Bagnall AM, Sowden AJ, Cornell JE, Mulrow CD, Ramirez G (2001). Interventions for the treatment and management of chronic fatigue syndrome: a systematic review. Journal of the American Medical Association 286, 1360-1368. 